\title{
BROADBAND GaAs pHEMT LNA DESIGN FOR T/R MODULE APPLICATION
}

\author{
Le Dai Phong,"*, Vu Duy Thong², Pham Le Binh ${ }^{3}$ \\ ${ }^{1}$ Institute of System Integration, Le Quy Don Technical University, \\ 236 Hoang Quoc Viet Street, Bac Tu Liem, Hanoi, Vietnam \\ ${ }^{2}$ Department of Electronics and Computer Engineering, VNU University of Engineering and \\ Technology, 144 Xuan Thuy Street, Cau Giay, Hanoi, Vietnam \\ ${ }^{3}$ Vimmics, 466/4 Le Quang Dinh, Binh Thanh, Ho Chi Minh city \\ "Email:phongld@mta.edu.vn
}

Received: 16 September 2015; Accepted for publication: 1 June 2016

\begin{abstract}
In this paper, a three stages monolithic low noise amplifier for $\mathrm{T} / \mathrm{R}$ module application is presented. This amplifier is fully integrated on $0.15 \mu \mathrm{m}$ GaAs pHEMT technology and achieves a wide bandwidth from 6 to $11 \mathrm{GHz}$. Within this band, the LNA has the minimum of $1.3 \mathrm{~dB}$ noise figure and over $25 \mathrm{~dB}$ small signal gain. The output third-order intercept point is over 30 $\mathrm{dBm}$ and the $1 \mathrm{~dB}$ compression point $\left(\mathrm{P}_{1 \mathrm{~dB}}\right)$ is $16 \mathrm{dBm}$ at the output.
\end{abstract}

Keywords: LNA; T/R Module; X-Band; MMIC; GaAs; radar; integrated circuit.

\section{INTRODUCTION}

Transmit/receive module (T/R module) is one of the most important elements in a radar system. A phased array antenna in a radar system uses thousands of such T/R modules. Figure 1 shows a block diagram of a T/R module. For the receiving function of T/R module, a low noise amplifier (LNA) is the key component that affects a lot of important system parameters such as noise figure (NF), gain, bandwidth (BW), spurious free dynamic range (SFDR), and spectral purity... The emerging in applications of radar systems, especially at X-band and Ku-band frequencies, necessitates wide frequency range, low noise, high gain, and high power T/R modules. Hence, a low noise, wideband, high gain, and high power LNA is highly demanded for next generation radar systems.

Recently, there are a lot of publications about X-band LNA. Some of them were designed on silicon substrate technology [1-3]. This technology can provide good noise figure and frequency performance with small dimension factors. However, some other crucial components in T/R module, such as power amplifier and switch, need to be developed with higher power and reliability that the silicon substrate technology cannot achieve. Gallium Arsenide (GaAs) technology, on the other hand, can provide high reliability and higher power density. The ref. [4] presents a 8 to $10 \mathrm{GHz}$ LNA on $0.25 \mu \mathrm{m}$ GaAs pHEMT with an output $\mathrm{P}_{1 \mathrm{~dB}}$ of $14 \mathrm{dBm}$. Besides, the LNA has a minimum noise figure of $1.4 \mathrm{~dB}$ and the gain of $29 \mathrm{~dB}$. In [5], the monolithic 
GaAs LNA achieves a very low noise figure of $0.5 \mathrm{~dB}$ and $30 \mathrm{~dB}$ gain. The frequency range of this LNA is, however, only from 7 to $10 \mathrm{GHz}$ and the output $\mathrm{P}_{1 \mathrm{~dB}}$ is $10 \mathrm{dBm}$.

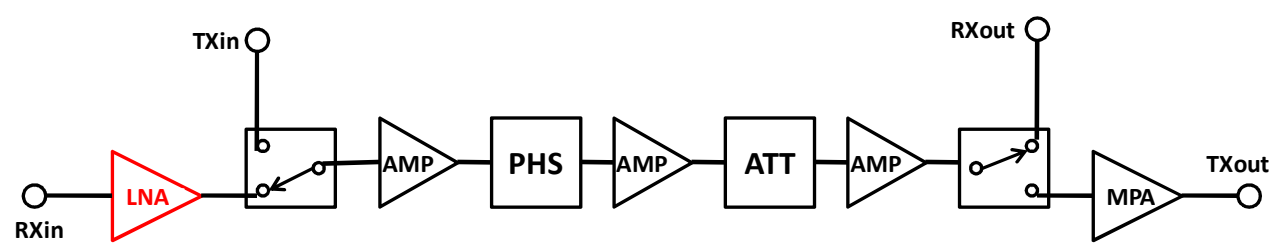

Figure 1. Block diagram of a T/R module.

This paper proposes a design of wideband, low noise, high gain, high power, and linearity monolithic LNA on $0.15 \mu \mathrm{m}$ pHEMT technology. The LNA achieves a bandwidth of 6 to 12 GHz. In this operating frequency band, the proposed design has the minimum NF of $1.3 \mathrm{~dB}$ and over $25 \mathrm{~dB}$ small signal gain. The output $1 \mathrm{~dB}$ compression point is $16 \mathrm{dBm}$ and the maximal third-order intercept point (OIP3) is $33 \mathrm{dBm}$.

\section{CIRCUIT DESIGN AND TECHNOLOGY}

\subsection{Devices technology and characteristic}

This LNA is designed on $0.15 \mu \mathrm{m}$ double recess GaAs Pseudomorphic High Electron Mobility Transistors (pHEMT) process from Win Semiconductor [6]. This process is built on 100 $\mu \mathrm{m}$ GaAs substrate and demonstrates good device level performance with $f_{t}$ of $90 \mathrm{GHz}$, power density of $860 \mathrm{~mW} / \mathrm{mm}$ at $29 \mathrm{GHz}$, more than $10 \mathrm{~dB}$ gain per transistor and $50 \%$ power added efficiency. The process exhibits high breakdown voltages of $16 \mathrm{~V}$ and therefore provides substantial operating margin for high reliability. It also allows a good minimum noise figure of about $0.5 \mathrm{~dB}$ at $10 \mathrm{GHz}$ for the $2 \times 75 \mu \mathrm{m}$ gate width transistor.

\subsection{LNA topology}

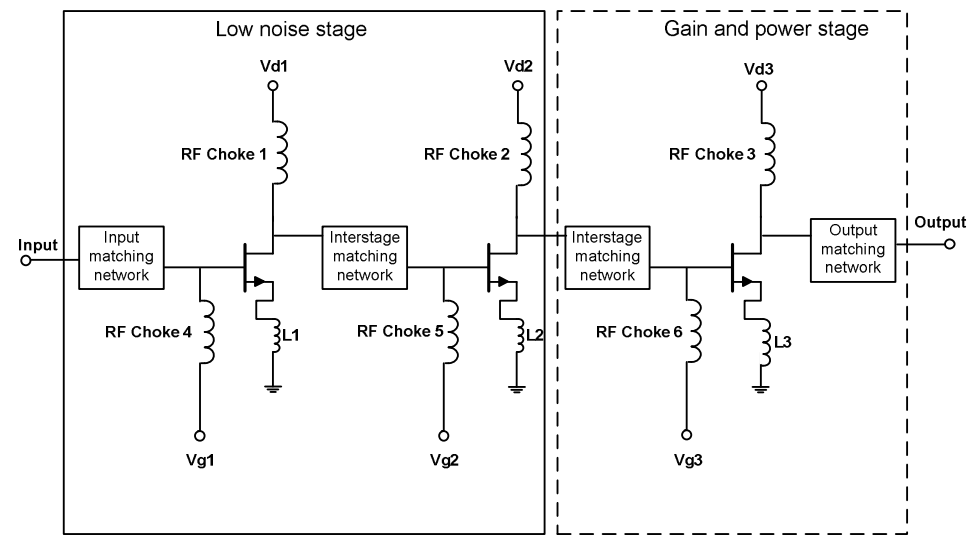

Figure 2. LNA topology.

Figure 2 shows the designed LNA topology. This LNA consists of three transistor stages in order to produce enough gain. The first two transistor stages are designed to have a low noise 
figure, whereas the last stage is optimized for gain, output power and stability. Choke inductors are used at all DC bias circuits to prevent radio frequency signal leakage. The LNA utilizes source degeneration matching technique with common source topology in order to achieve good return loss and low noise matching over a wide bandwidth simultaneously.

\subsection{Design for low noise figure}

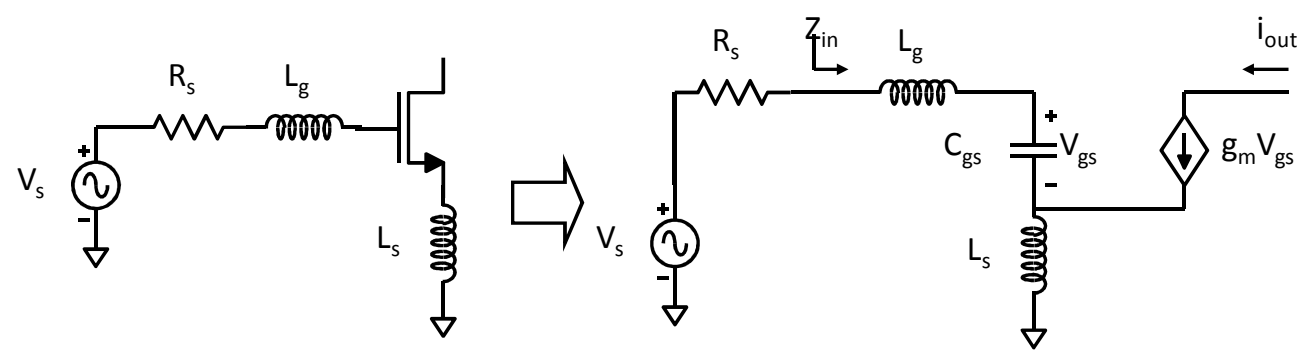

Figure 3.Inductive source degeneration topology and its small signal equivalent circuit.

As we mentioned in the previous section, the first two stages is matched for low noise figure. There are several matching techniques such as resistive termination, series-shunt feedback, input matched LNA (without degeneration inductor)... The first two techniques allow very good return loss. However, they are still noisy due to resistive noise source and attenuate signal. The input matched LNA technique delivers better noise figure matching but it's hard to achieve good return loss at the same time. In [7], good return loss and noise performance can be achieved simultaneously by using inductive degeneration technique which has topology shown in Figure 3. From its small signal equivalent circuit, the input impedance $\mathrm{Z}_{\text {in }}$ is calculated

and the noise figure is

$$
Z_{i n}=s\left(L_{g}+L_{s}\right)+\frac{1}{s C_{g s}}+\frac{g_{m} L_{s}}{C_{g s}}
$$

$$
\left.N F\right|_{\omega \approx \omega_{0}}=1+\gamma \frac{4\left(g_{m} L_{s}\right)^{2}}{R_{s} g_{m}} \frac{1}{C_{g s}\left(L_{g}+L_{s}\right)}=1+\frac{4 \gamma L_{s}}{L_{g}+L_{s}}
$$

where $\gamma$ is empirical constant and equals $2 / 3$ for long channel. (1) and (2) show that good return loss and noise matching can be obtained simultaneously by having large $L_{g}$ and choosing appropriate $L_{s}$. Nevertheless, $L_{s}$ should be selected carefully, because available gain is reduced with large $L_{s}$. In the first two stages of this design, the source degeneration inductor $L_{s}$ is selected about $0.5 \mathrm{nH}$. [7] also states that a possible minimum noise factor for a device, $F_{\min }$, is only achieved when a particular reflection coefficient, $\Gamma_{\mathrm{s}}=\Gamma_{\mathrm{opt}}$ is presented to the input

$$
F=F_{\min }+\frac{4 r_{n}\left|\Gamma_{S}-\Gamma_{o p t}\right|^{2}}{\left(1-\left|\Gamma_{S}\right|^{2}\right)\left(1+\Gamma_{o p t}\right)^{2}}
$$

where $\mathrm{F}$ is the noise factor of a two port network; $F_{\text {min }}, r_{n}, \Gamma_{\text {opt }}$ are noise parameters giving by the foundry or measured; $\Gamma_{\mathrm{s}}$ is the reflection coefficient at the input.

Therefore, after selecting $L_{s}$, the impedance of $\Gamma_{\mathrm{s}}=\Gamma_{\mathrm{opt}}$ is searched by doing source-pull simulation at the gate of transistor. For this design, the impedance of $120+\mathrm{j} 145 \Omega$ is found and the input matching network is optimized near this optimum noise matching impedance. The gate 
width of the transistors in the first and second stages is $150 \mu \mathrm{m}$. The transistors are biased at $\mathrm{V}_{\mathrm{d} 1}=$ $\mathrm{V}_{\mathrm{d} 2}=2 \mathrm{~V}$ and $\mathrm{V}_{\mathrm{g} 1}=\mathrm{V}_{\mathrm{g} 2}=-0.8 \mathrm{~V}$ with the drain current $\mathrm{I}_{\mathrm{d} 1}=\mathrm{I}_{\mathrm{d} 2}=22 \mathrm{~mA}$.

\subsection{Design of the third stage}

Unlike the first two stages, the third stage of this LNA is designed for gain, output power and linearity. In order to have high output power and linearity, the bias point of this stage is moved to $\mathrm{V}_{\mathrm{d} 3}=5 \mathrm{~V}$ and $\mathrm{V}_{\mathrm{g} 3}=-0.6 \mathrm{~V}$ for the drain current $\mathrm{I}_{\mathrm{d} 3}=37 \mathrm{~mA}$. The total gate width of this stage is also $150 \mu \mathrm{m}$. In this stage, a very small source degeneration inductor is used to enhance the stability of the whole circuit. Besides, this inductor also decreases third order intermodulation distortion (IMD3) and helps to improve the linearity as discussed in [8]. The outputmatching network is designed to balance between a good wideband $S_{22}$, flat gain and high output power.

\section{THE LNA PROTOTYPE AND EXPERIMENTAL RESULTS}

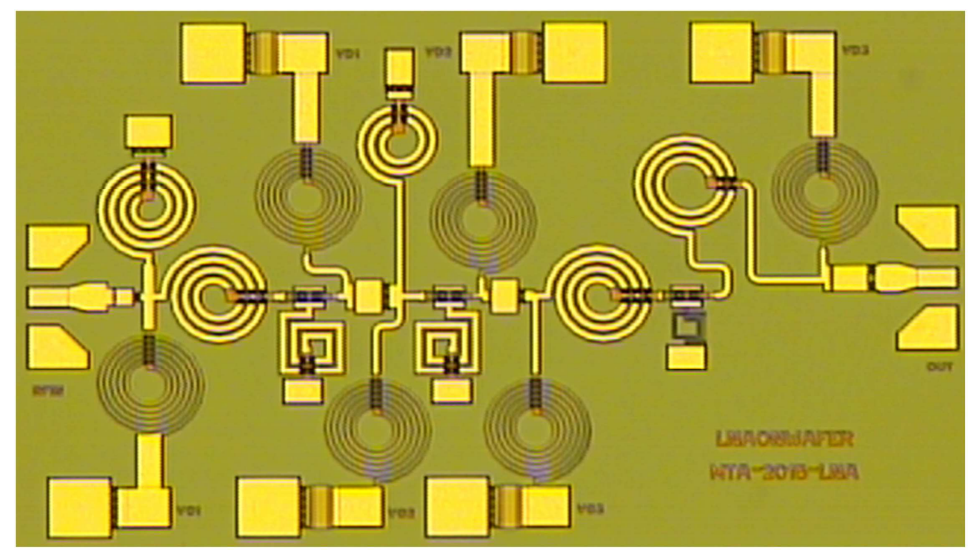

Figure 4. LNA chip photograph.

Figure 4 is the picture of the fabricated LNA chip. The dimension of the LNA die is 1.2 $\mathrm{mm}$ by $2.1 \mathrm{~mm}$. At the LNA's input and output, ground-signal-ground (GSG) pads are placed for on-wafer measurement. Gate and drain of each transistor are connected to DC pads allowing to adjust bias point at each stage independently. At each DC pads, a small resistor and a bypass capacitor are attached to ensure for the stability and reliability. The coupling effects and parasitic of the layout are predicted by using electromagnetic simulator AXIEM of Microwave Office AWR [9]. As we can see in Figure 5, the measured small signal s-parameters of the LNA show that the operating frequency is from 6 to $11 \mathrm{GHz}$ with over $25 \mathrm{~dB}$ small signal gain $\mathrm{S}_{21}$. The input return loss $S_{11}$ and output return loss $S_{22}$ are better than $6 \mathrm{~dB}$ in this band. The measured noise figure over operating frequency range is illustrated in Figure 6. The LNA has the noise figure of about $1.3-2 \mathrm{~dB}$ for the frequencies from 5.7 to $12 \mathrm{GHz}$. Figure 7 shows the large signal simulation of the LNA at $10 \mathrm{GHz}$. From Figure 7, the $\mathrm{P}_{1 \mathrm{~dB}}$ is at $16 \mathrm{dBm}$ output power and $-12.4 \mathrm{dBm}$ input power. The OIP3 of this circuit is found by feeding 2-tones signal, which are separated by $10 \mathrm{MHz}$ at the input. Figure 8 shows that the OIP3 is greater than $30 \mathrm{dBm}$ from 8 to $12 \mathrm{GHz}$ and has maximal OIP3 of $33 \mathrm{dBm}$ at $10 \mathrm{GHz}$. Table 1 summarizes the performance of this design and compares with some previous published GaAs LNAs. 


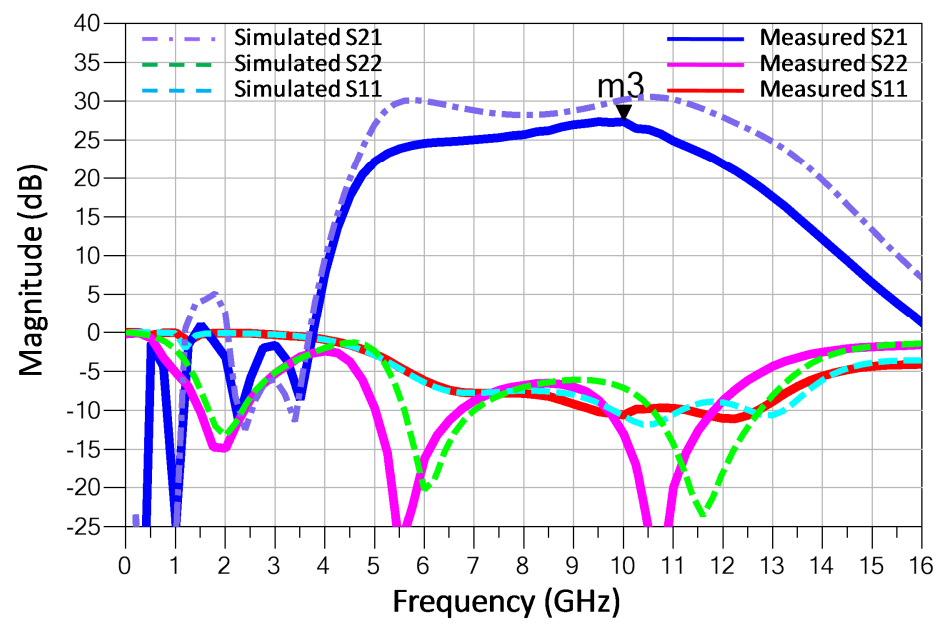

Figure 5.Simulatedand measured small signal s-parameters of LNA.

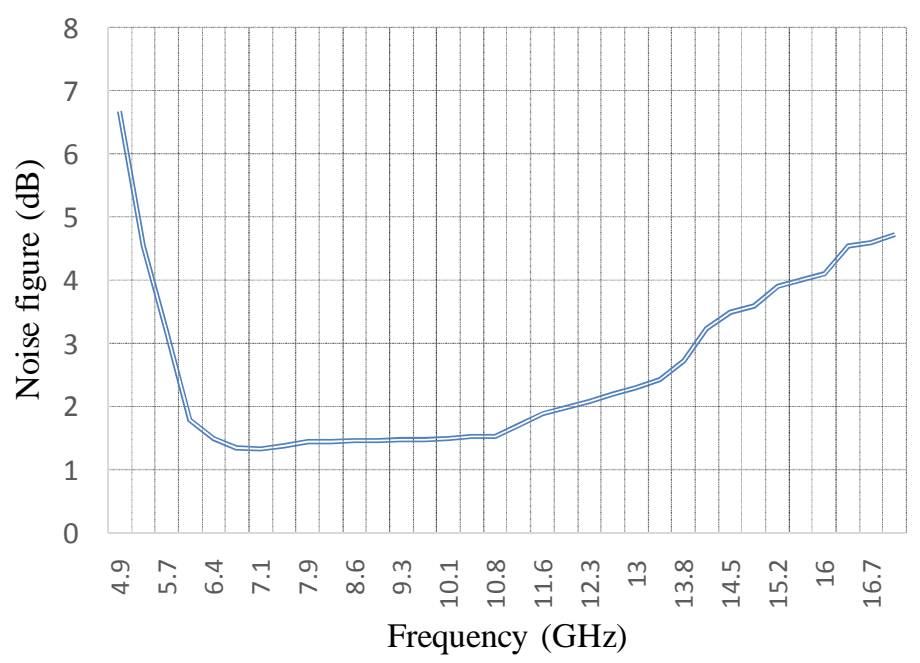

Figure 6. Measured noise figure.

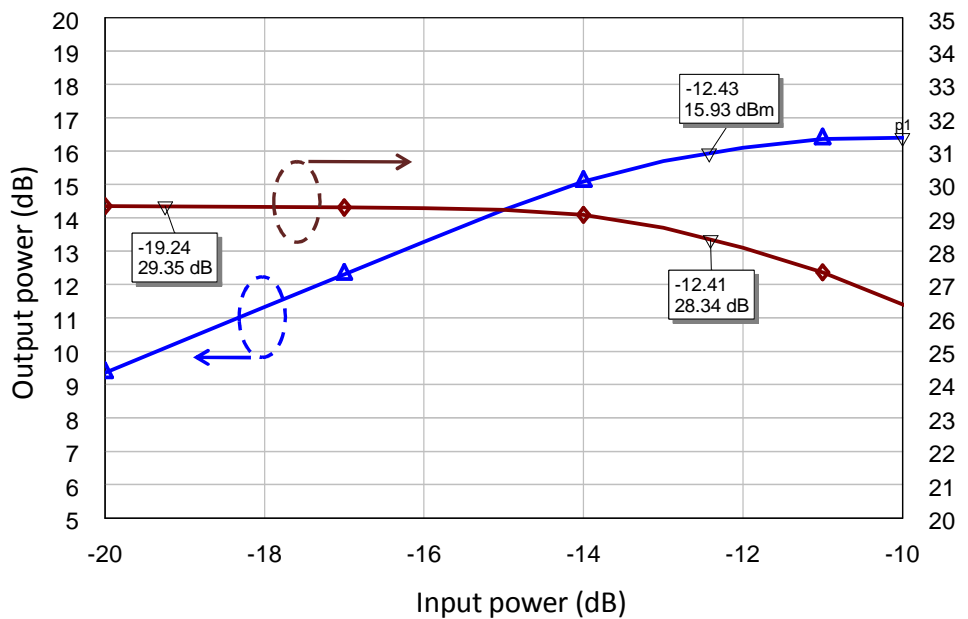

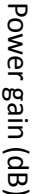

Figure 7.Output power versus Input power at $10 \mathrm{GHz}$. 


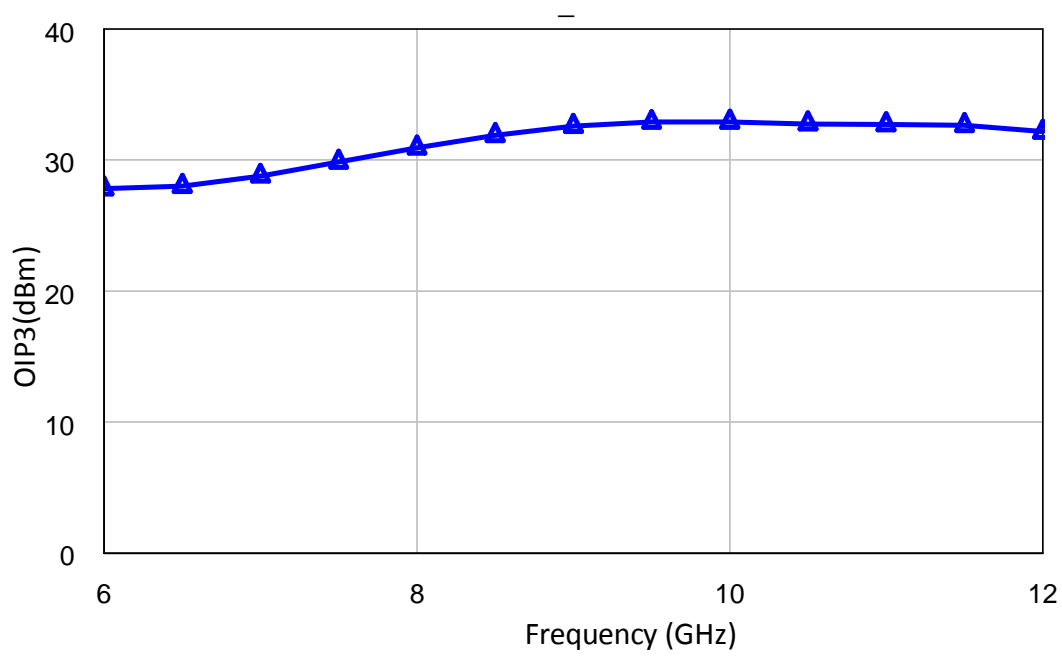

Figure 8.Output third-order intercept point versus frequency.

Table 1. LNAs comparison.

\begin{tabular}{|c|c|c|c|c|c|c|c|}
\hline & $\begin{array}{c}\text { Frequency } \\
(\mathrm{GHz})\end{array}$ & Gain $(\mathrm{dB})$ & $\mathrm{P}_{1 \mathrm{~dB}}(\mathrm{dBm})$ & $\begin{array}{c}\text { OIP3 } \\
(\mathrm{dBm})\end{array}$ & $\begin{array}{c}\text { NF } \\
(\mathrm{dB})\end{array}$ & $\begin{array}{c}\text { Chip Area } \\
\left(\mathrm{mm}^{2}\right)\end{array}$ & Process \\
\hline$[10]$ & $6-14$ & 20 & 12 & 24 & 1.3 & $2.05 \times 1.2$ & GaAs \\
\hline$[11]$ & $5-11$ & 27 & 13 & 25 & 1.4 & $2.3 \times 1.35$ & GaAs \\
\hline$[12]$ & $7-11$ & 26 & 1 & N/A & 1 & $1.5 \times 1$ & GaAs \\
\hline$[13]$ & $8-12$ & 30 & 10 & N/A & 1.5 & $2.5 \times 1.5$ & GaAs \\
\hline$[14]$ & $3.2-14.7$ & 34 & N/A & N/A & 1.3 & $2.5 \times 1.5$ & GaAs \\
\hline This work & $6-11$ & 25 & 16 & 33 & 1.3 & $2.1 \times 1.2$ & GaAs \\
\hline
\end{tabular}

\section{CONCLUSIONS}

A wideband X-band LNA integrated circuit have been designed using $0.15 \mu \mathrm{m}$ GaAs pHEMT technology. In the frequency band from 6 to $11 \mathrm{GHz}$, the LNA achieves excellent performance with more than $25 \mathrm{~dB}$ gain and $1.3-2 \mathrm{~dB}$ noise figure. The output $1 \mathrm{~dB}$ compression power is $16 \mathrm{dBm}$ and third-order intercept point is greater than $30 \mathrm{dBm}$. The LNA occupies $2.52 \mathrm{~mm}^{2}$ and is unconditional stable.

Acknowledgment. This work is the results of the research KC01.19/11-15 which was sponsored by MOST. The authors would like to thank National Science and Technology Program of Vietnam; Professor Anh-Vu Pham, University of California, Davis, USA for dedicated contribution in this project.

\section{REFERENCES}

1. Jeng-Han Tsai, Wang-Long Huang, Cheng-Yen Lin, and Ruei-An Chang - An X-band low-power CMOS low noise amplifier with transformer inter-stage matching networks, Proc. 44th European Microwave Conference (EuMC) (2014) 1468-1471. 
2. Kanar T. and Rebeiz G. M. - X- and K-Band SiGe HBT LNAs With 1.2- and 2.2-dB Mean Noise Figures, IEEE Trans. Microwave Theory Tech. 62 (10) (2014) 2381-2389.

3. Thrivikraman T. K., Jiahui Yuan, Bardin J. C., Mani H., Phillips S. D., Wei-Min Lance Kuo, Cressler J. D., and Weinreb S. - SiGe HBT X-Band LNAs for Ultra-Low-Noise Cryogenic Receivers, IEEE Microwave and Wireless Components Letters 18 (7) (2008)4 76-478.

4. Giannini F., Limiti E., Serino A., and Dainelli V. - A medium-power low-noise amplifier for X-band applications, Proc.34th European Microwave Conference 1 (2004) 37-39.

5. Heins M. S., Carroll J. M., Kao M., Delaney J., and Campbell C. F. - X-band GaAs mHEMT LNAs with $0.5 \mathrm{~dB}$ noise figure, IEEE MTT-S International Microwave Symposium Digest 1 (2004) 149-152.

6. [Online]. Available: http://www.winfoundry.com/en_US/support.aspx?sn=8

7. Behzad Razavi, "RF Microelectronics", Prentice Hall, 2nd edition, 2011.

8. Myoung-Gyun Kim and Tae-Yeoul Yun - Anaysis and design of linearity improved mixer using third-order transconductance cancellation, Proc.3rd IEEE International Conference on Network Infrastructure and Digital Content (IC-NIDC)(2012)652-655.

9. [Online]. Available: http://www.awrcorp.com/

10. [Online]. Available: http://www.triquint.com/products/p/TGA2511

11. [Online]. Available: http://www.custommmic.com/CMD229_Low_Noise_Amplifier/

12. Bhaumik S. and Kettle D. - Broadband X-band low noise amplifier based on $70 \mathrm{~nm}$ GaAs metamorphic high electron mobility transistor technology for deep space and satellite communication networks and oscillation issues, IET Microwaves, Antennas \& Propagation 4 (9) (2010) 1208-1215.

13. Arykov V. S., Barov A. A., Velikovskiy L. E., and Kondratenko A. V. - X-band GaAs pHEMT MMIC low-noise amplifier, Proc. 21th International Crimean Conference on Microwave and Telecommunication Technology (CriMiCo) (2011)159-160.

14. Yunshan Wang, Chau-Ching Chiong, Ji-Kang Nai, and Huei Wang - A high gain broadband LNA in GaAs $0.15 \mu \mathrm{m}$ pHEMT process using inductive feedback gain compensation for radio astronomy applications, Proc. IEEE International Symposium on Radio-Frequency Integration Technology (RFIT) (2015) 79-81. 